\title{
VASODILATING EFFECT OF CRUDE PLATYCODIN IN ANESTHETIZED DOGS
}

\author{
Hitoshi KATO, Shoshi SUZUKI, Kenichiro NAKAO, Eun B. LEE \\ and Keijiro TAKAGl \\ Department of Chemical Pharmacology, Faculty of Pharmaceutical Sciences, \\ University of Tokyo, Bunkyo-ku. Tokyo. Japan
}

Accepted March 31, 1973

\begin{abstract}
The effects of crude platycodin on the coronary and hindquarter vascular beds in anesthetized dogs were studied and compared with those of glyceryl trinitrate, papaverine and Saponin (E. Merck AG.). Crude platycodin in doses of 200 to 800 $\mu g$ i.a. dose-dependently reduced coronary and hindquarter vascular resistances and the potency was comparable to that of papaverine. Crude platycodin, $4 \mathrm{mg} / \mathrm{kg}$ i.y., also caused an increase in coronary and hindquarter blood flows, concomitantly with a transient systemic hypotension. The vasodilating effect of crude platycodin appears to be direct and non-specific on the peripheral blood vessels as in the case of glyceryl trinitrate or papaverine.
\end{abstract}

A Chinese medicine "Jiegeng" (Japanese name: Kikyo), the roots of Platycodon grandiforum A. DC., has been frequently administered to patients with cough and/or bronchitis as an expectorant. Crude platycodin, a saponin fraction separated from the methanolic extracts of the roots (1) has been shown to have anti-inflammatory, anti-ulcer, vasodilating and expectorant activitics in experimental animals (2-4). The marked inhibitory effect of crude platycodin on gastric secretion and experimental ulcerations have also been reported (5).

In the present studies, the effects of crude platycodin on peripheral circulation including the coronary and hindquarter vascular beds in anesthetized dogs were investigated and compared with those of glyceryl trinitrate, papaverine and Saponin (E. Merck AG.).

\section{MATERIALS AND METHODS}

Male mongrel dogs weighing 10 to $15 \mathrm{~kg}$ were anesthetized with sodium pentobarbital, $35 \mathrm{mg} / \mathrm{kg}$ i.v. The trachea was cannulated and the animals were artificially ventilated with room air using a positive pressure respirator (Natsume, KN-50). The chest was opened at the left fourth intercostal space, and the heart was exposed. After heparinization ( $500 \mathrm{unit} / \mathrm{kg}$ i.v.), the left anterior descending coronary artery was proximally ligated, distally cannulated and perfused with blood led from the left common carotid artery through polyvinyl tubing and an electromagnetic flowmeter (Nihon Kohden, MF-2). The right lemoral arterial blood pressure was obtained via a pressure transducer (Nihon Kohden, MPU-0.5) and recorded together with coronary blood flow on a polygraph (Nihon Kohden, RM-150). 
For the measurement of femoral blood flow, pentobarbitalized and heparinized dogs as mentioned above were utilized. The left femoral artery was exposed and cannulated in its proximal portion, the blood led from the cannula was streamed into another cannula inserted into the distal portion of the artery through polyvinyl tubing and an electromagnetic flowmeter.

The following parameters were calculated: coronary vascular resistance $=$ = mean femoral arterial blood pressure $(\mathrm{mmHg}) /$ mean coronary blood flow $(\mathrm{m} / \mathrm{min})$, and hindquarter vascular resistance-mean femoral blood pressure $(\mathrm{mmHg}) /$ mean femoral blood flow (mi/min).

Each drug solution having no influence on systemic blood pressure was injected close to the perfusing artery in a volume of $0.4 \mathrm{ml}$ in $10 \mathrm{sec}$.

In some experiments, the effects of intravenously administered agents on the coronary and hindquarter vascular beds were examined. The blood flow through the left anterior descending coronary artery or the left femoral artery was measured with a flow probe (internal diameter: 2 to $3 \mathrm{~mm}$, Nihon Kohden) positioned around the corresponding vessel. The flow probe signals were fed into an electromagnetic flowmeter (Nihon Kohden, MF5), and recorded together with femoral arterial blood pressure and heart rate obtained via a cardiotachometer (Nihon Kohden, RT-2) triggered by the femoral arterial pulse on a polygraph. Each drug solution was injected into the left cephalic vein through an inserted catheter and was fyushed with $1 \mathrm{ml}$ of physiological saline.

Crude platycodin, readily soluble in water and a pale-yellowish brown powder with a bitter taste, mainly consisted of saponins, and was prepared by Akiyama et al. (1). Glyccryl trinitrate $(0.01 \%$ aqueous solution, Nihon Kayaku Co.). papaverine hydrochloride (Iwaki Seiyaku Co.), Saponin (E. Merck AG.), isoproterenol hydrochloride (Kaken Chemical Co.), histanine dihydrochloride (Wako Pure Chemicals Co.), acetylcholine chloride (Daiichi Seiyaku Co.), atropine sulfate (Wako Pure Chemicals Co.), diphenhydramine hydrochloride (Tokyo Kasei Co.), propranolol hydrochloride (Sumitomo Chemical Co.) and crude platycodin were freshly dissolved in physiological saline in the required concentrations. The dose of the drugs was expressed in terms of the salt.

\section{RESULTS}

1. Effect on the coronary vascular bed

The effects of crude platycodin in doses of 200,400 and $800 \mu \mathrm{g}$ i.a. on the coronary vascular bed were examined in comparison with those of glyceryl trinitrate, 2,4 and $8 / 2 \mathrm{~g}$ i.a., papaverine and Saponin, 200, 400 and $800 \mathrm{~kg}$ i.a. All these agents exerted a dosedependent vasodilating effect on the coronary vascular bed. Typical data are shown in Fig. 1. In ten separate experiments performed on different dogs, the maximum effects of crude platycodin, 200, 400 and $800 \mu$ i.a., were $53.5-3.0$ (S.E.), $64.1\lfloor 1.9$ and 65.5 $1.7 \%$ decreases in coronary vascular resistance, respeclively, as shown in Fig. 2. These values were significantly greater than those of Saponin $(p<0.05)$, but not significant from those of papaverine in each dose tested, although the mean values obtained from the ad- 


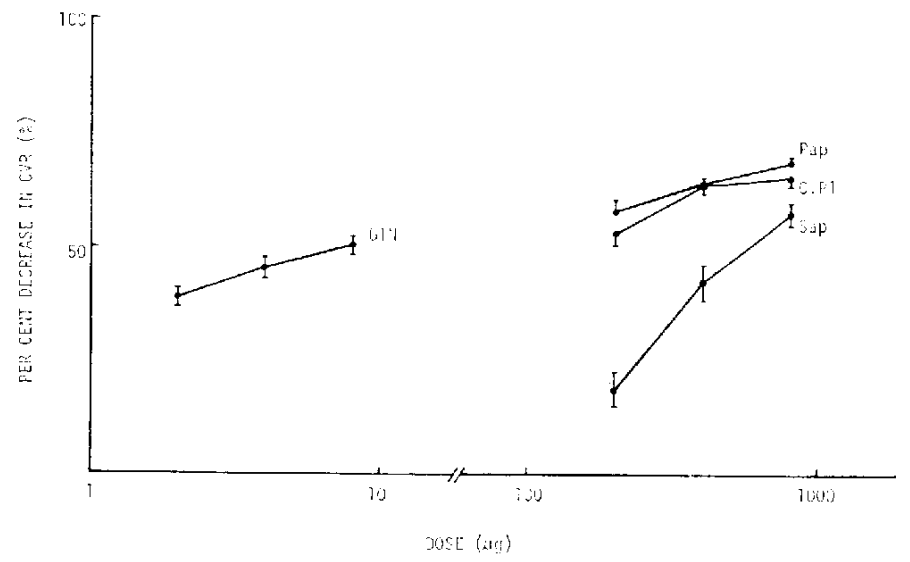

FIG. 1. Effects of crude platycodin, glyceryl trinitrate, papaverine and Saponin on coronary vascular resistance in ancsthetized dogs. GTN : glyceryl trinitrate, Pap : papaverine, C.Pl : crude platycodin, and Sap : Saponin. Abscissa : dose of the agents in $/ \mathrm{g}$ i.a. and ordinate : per cent decrease in coronary vascular resistance. Each point represents the mean value obtained from ten separate experiments performed on different dogs, and vertical bars represent standard errors.
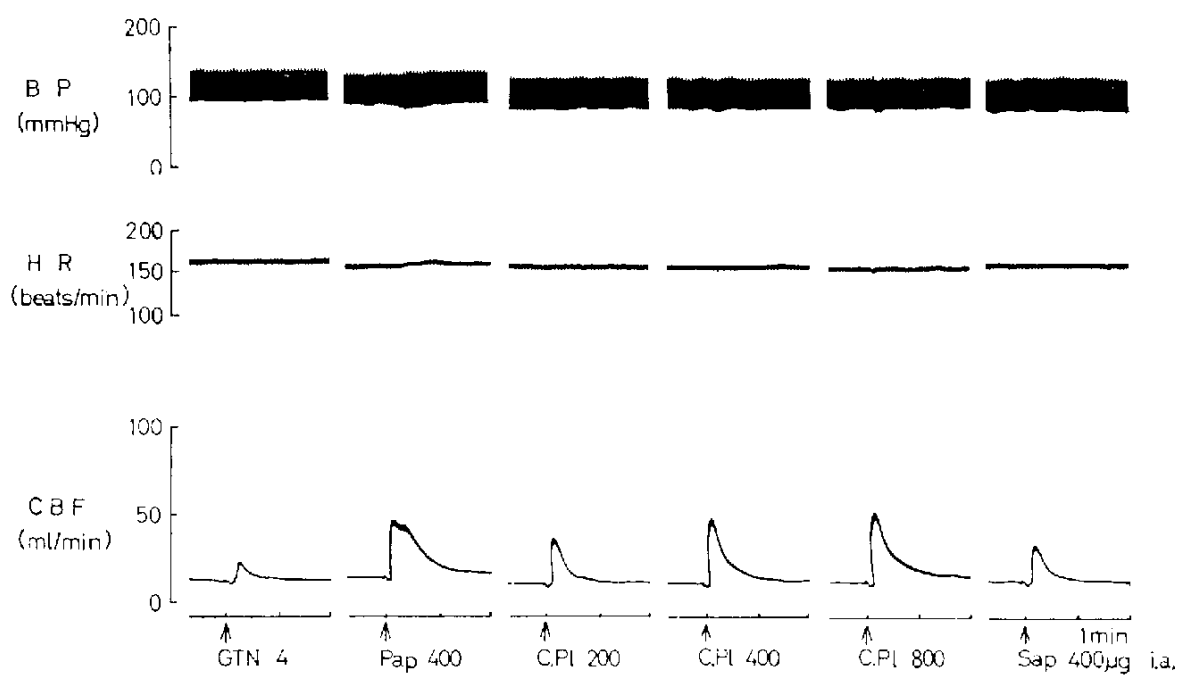

FIG. 2. Effects of intra-arterially administered crude platycodin, glyceryl trinitrate, papaverine and Saponin on coronary blood flow in an anesthctized dog. BP : femoral arterial blood pressure, HR : heart rate, CBF : coronary blood flow, GTN : glyceryl trinitrate, Pap : papaverine, C.PI : crude platycodin, and Sap : Saponin. Each drug solution was injected at the arrow.

ministration of crude platycodin were less than those of papaverine. The potency of glyccryl trinitrate could not be compared with these agents, as only a low concentration had been prepared.

In three dogs, crude platycodin, $4 \mathrm{mg} / \mathrm{kg}$ i.v., also caused an increase in coronary blood flow, as shown in Fig. 3. Saponin, $4 \mathrm{mg} / \mathrm{kg}$ i.v., exerted a similar effect but less 

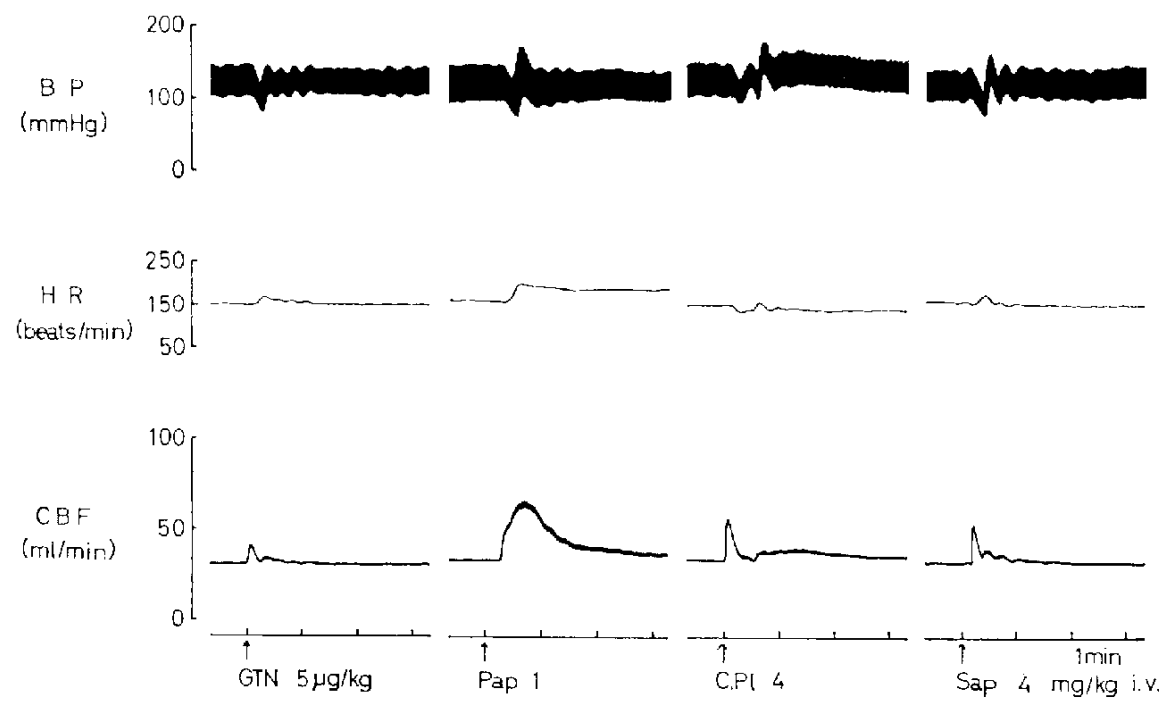

Fici. 3. Effects of intravenously administered crude platycodin, glyceryl trinitrate, papaverine and Saponin on coronary blood flow in an anesthetized dog. Notations as in Fig. 2.

potent than that of crude platycodin. Papaverine, $1 \mathrm{mg} / \mathrm{kg}$ i.v., induced a more marked and persistent increase in coronary blood flow than crude platycodin. Glyceryl trinitrate, $5 \mu \mathrm{g} / \mathrm{kg}$ i.v., had little effect on coronary blood flow.

2. Effect on the hindquarter vascular bed

Close-arterially administered crude platycodin caused an increase in femoral blood flow as in the case of coronary blood flow mentioned above. Typical data are shown in

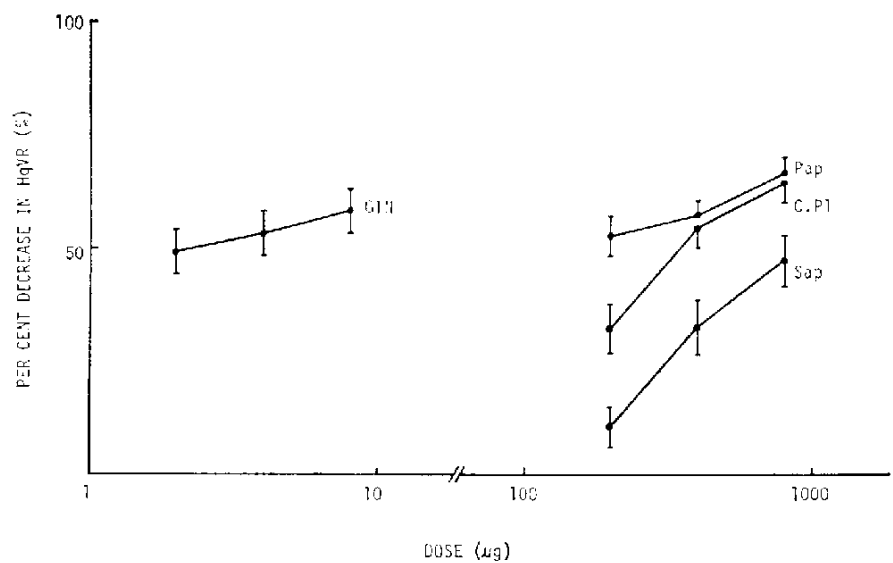

FIG. 4. Effects of crude platycodin, glyceryl trinitrate, papaverine and Saponin on hindquarter vascular resistance in anesthetized dogs. GTN : glyceryl trinitrate, Pap : papaverine, C.Pl : crude platycodin, and Sap : Saponin. Abscissa : dose of the agents in $;$ g i.a. and ordinate : per cent decrease in hindquarter vascular resistance. Each point represents the mean value obtained from ten separate experiments performed on different dogs, and vertical bars represent standard errors. 

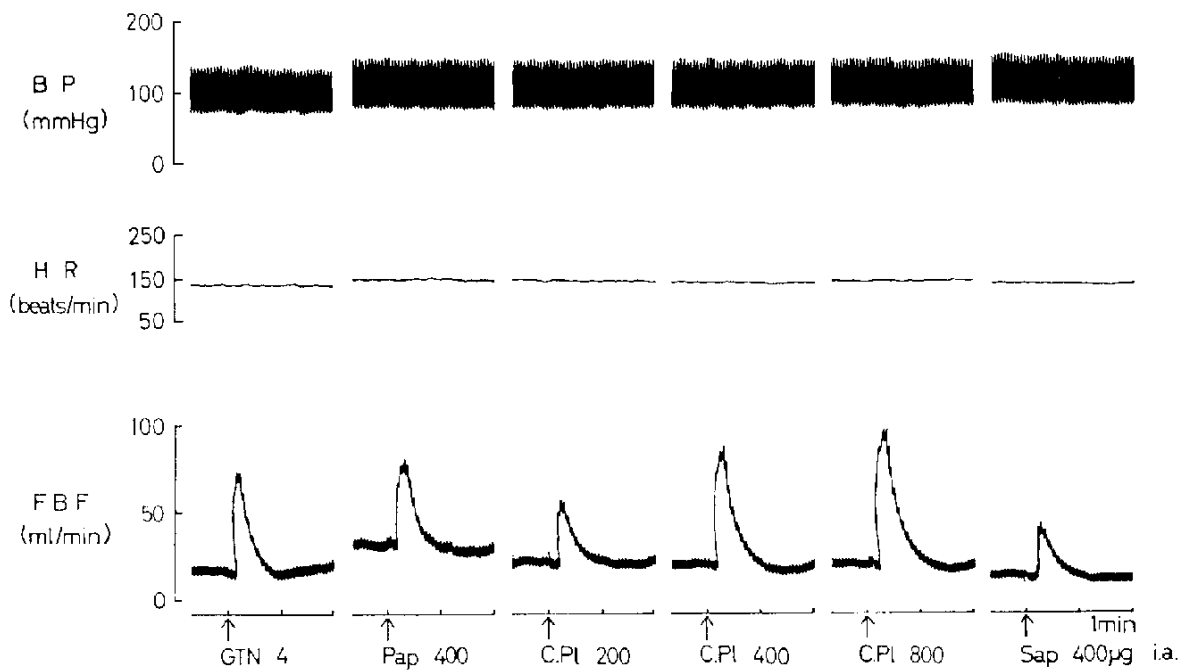

FIG. 5. Effects of intra-arterially administered crude platycodin, glyceryl trinitrate, papaverine and Saponin on femoral blood flow in an anesthetized dog. BP: femoral arterial blood pressurc, HR : heart rate, FBF : femoral blood flow, GTN : glyceryl trinitrate, Pap : papaverine, C.Pl : crude platycodin, and Sap : Saponin. Each drug solution was injected at the arrow.
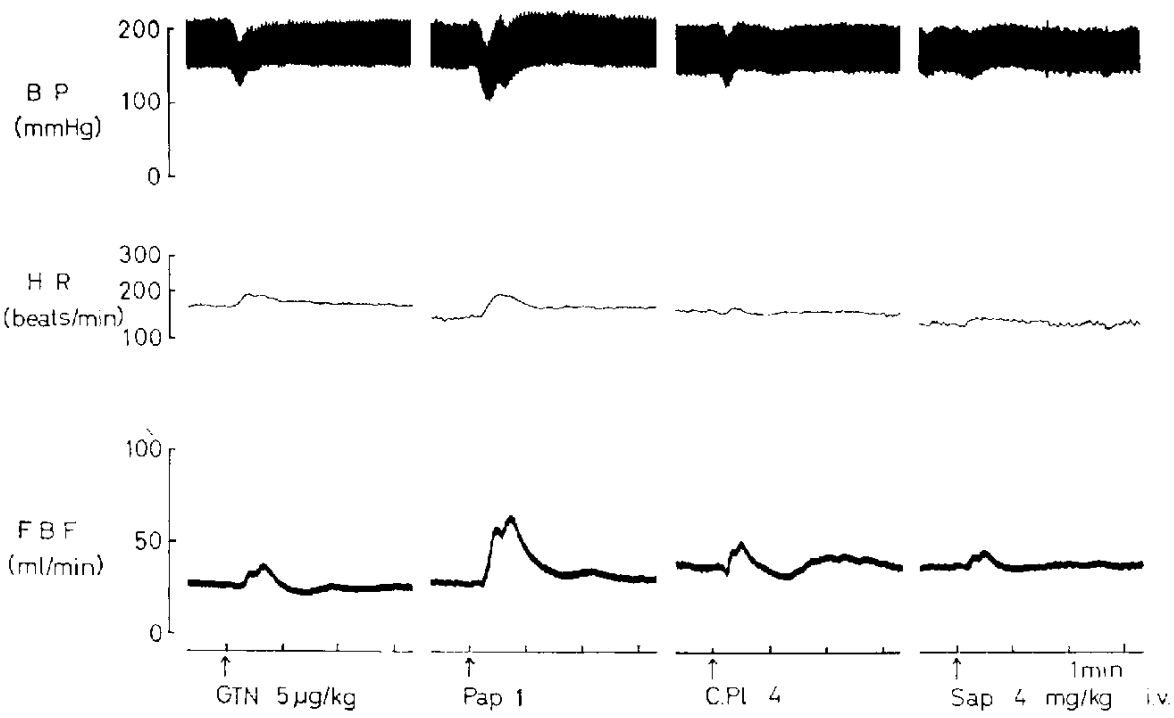

FIG. 6. Effects of intravenously administered crude platycodin, glyceryl trinitrate, papaverine and Saponin on femoral blood flow in an ancsthetized dog. Notations as in Fig. 5.

Fig. 4. Crude platycodin in doses of 200,400 and $800 \mu$ g i.a. showed $31.9 \pm 5.0$ (S.E.), $54.5 \pm 4.2$ and $64.3+3.9 \%$ decreases in hindquarter vascular resistance $(\mathrm{N}=10)$, respectively, as shown in Fig. 5. These changes were significantly greater than those of Saponin $(p<0.05$ ), but not significant from those of papaverine in each dose tested. The mean 
values of per cent decrease in hindquarter vascular resistance obtained from the administration of crude platycodin were, however, less than those of papaverine when the same dose was administered.

In three dogs, crude platycodin, $4 \mathrm{mg} / \mathrm{kg}$ i.v., also exerted an increase in femoral blood flow, concomitantly with a transient fall in systemic blood pressure, as shown in Fig. 6. Saponin, $4 \mathrm{mg} / \mathrm{kg}$ i.v., and glyceryl trinitrate, $5 \mu \mathrm{g} / \mathrm{kg}$ i.v., showed a similar effect but less potent than that of crude platycodin. Papaverine, $1 \mathrm{mg} / \mathrm{kg}$ i.v., produced a more marked increase in femoral blood flow than the other three agents.

\section{Mechanism intolved in vasodilating effect}

To study the mechanism involved in the vasodilating effect of crude platycodin on the coronary and hindquarter vascular beds, the following experiments were performed.

Influcnce of treatment with atropine, $2 \mathrm{mg} / \mathrm{kg}$, diphenhydramine, $3 \mathrm{mg} / \mathrm{kg}$, or propranolol, $0.5 \mathrm{mg} / \mathrm{kg}$ i.v., on the vasodilating effect of crude platycodin, $400 \mu \mathrm{g}$ i.a., administered into the coronary or hindquarter vascular bed was observed and compared with those of glyceryl trinitrate, $4 \mu \mathrm{g}$, papaverine, $400 \mu \mathrm{g}$, and Saponin, $400 \mu \mathrm{g}$ i.a. Each corresponding blocking effect of these blocking agents was verified by the administrations of acetylcholine, $0.1 \mu \mathrm{g}$, histamine, $0.1 \mu \mathrm{g}$, and isoproterenol, $0.1 \mu \mathrm{g}$ i.a., respectively. Crude platycodin, $400 \mathrm{gg}$ i.a., induced 50 to $60 \%$ increase in coronary vascular resistance in six dogs, and no significant change after treatment with each blocking agent was observed, as is shown in Fig. 7. In the case of glyceryl trinitrate, papavcrine and Saponin, no influcnce of treatment with atropine, diphenhydramine or propranolol was observed. Similar results were obtained from experiments on hindquarter vascular resistance in six animals, as shown in Fig. 8.

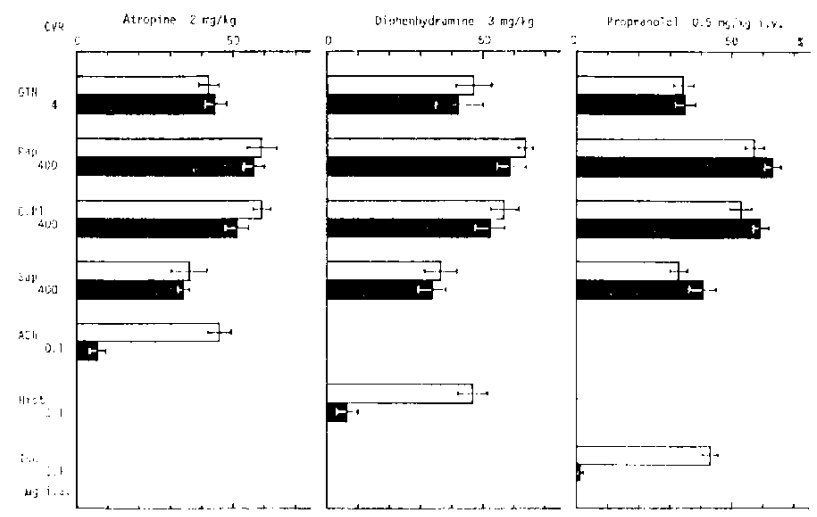

Fig. 7. Influence of atropine, diphenhydramine and propranolol on changes in coronary vascular resistance induced by crude platycodin, glyceryl trinitrate, papaverine and Saponin in anesthetized dogs. GTN : glyceryl trinitrate, Pap : papaverine, C.PI : crude platycodin, Sap : Saponin, ACh : acetylcholine, Hist : histamine, and Iso : isoproterenol. Abscissa : per cent dccrease in coronary vascular resistance. White column : before treatment with each blocking agent, and black column : after treatment. Each bar represents standard error of mean value obtained from six separate experiments performed on different dogs. 


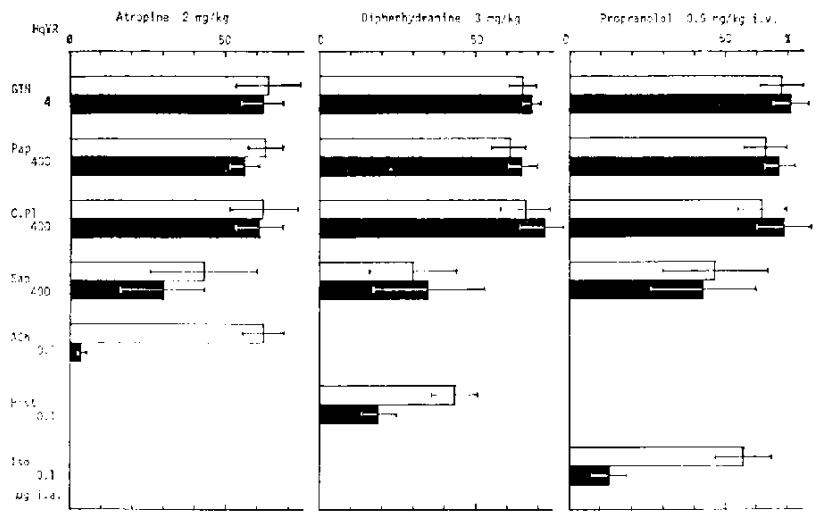

FIG. 8. Influence of atropine, diphenhydramine and propranolol on changes in hindquarter vascular resistance induced by crude platycodin, glyceryl trinitrate, papaverine and Saponin in anesthetized dogs. Notations as in Fig. 7, except abscissa : per cent decrease in hindquarter vascular resistance.

\section{DISCUSSION}

Crude platycodin is a crude saponin fraction isolated from platycodi radix and apparently contains a specific component of this oriental plant which has been used as a Chinese medicine (1).

As the result of a semi-quantitative trial carried out in order to clarify the therapeutic effects of platycodi radix in Chinese medicine, platycodi radix was shown to be mainly prescribed for patients with various inflammatory diseases (6). This plant has also been uscd for its expectorant, analgesic, antipyretic, hypotensive and antitussive effects. Furthermore, sedative, anti-ulcer, antigastritic and anti-allergic effects of the plant have occasionally been observed.

On the other hand, crude platycodin has revealed anti-inflammatory, anti-ulcer, vasodilating and expectorant effects as main pharmacological activities in experimental animals. Analgesic, antipyretic, antitussive and anticholinergic effects have also been observed (2-4).

In the present studies, close-arterially administered crude platycodin showed a vasodilating effect on coronary and hindquarter vascular beds autoperfused in pentobarbitalized dogs. From the results of per cent decreases in both peripheral vascular resistances, the vasodilating potency of crude platycodin in doses used was comparable to that of papaverine, and significantly superior to that of Saponin (E. Merck AG.). Furthermore, crude platycodin clearly showed increases in coronary and femoral blood flows, even when administered intravenously.

It is widely recognized that coronary blood flow may be influenced by a change in cardiac contractile force. Crude platycodin, even in a rclatively high concentration $(1 \times$ $10^{-3}$ to $3 \times 10^{-3} \mathrm{~g} / \mathrm{ml}$ ), has been shown to cause only a mild depression of contractile force in isolated guinea pig atria (4). For this reason, an increase in coronary blood flow induced by crude platycodin in doses utilized herein, does not appear to be due to a change in contractile force, although this force was not measured in the present studies. 
A relatively significant hemolytic activity of crude platycodin has been observed: crude platycodin is 1.2 times stronger than Saponin (E. Merck AG.) in hemolytic index (2). Whether or not the vasodilating effect observed is influenced by hemolytic activily is unclear.

Although experiments on vascular beds other than the coronary and hindquarter have not been carricd oul, crude platycodin appears to have no speciffe vasodilating effect on a particular vascular bed, sinee the agent showed the activity in both coronary and hindquarter vascular beds in almost the same degree.

To determine the vasodilating mechanism of crude platycodin, some blocking agents, including atropine, diphenhydramine and propranolol, were utilized. Since there was no influence by these blocking agents on the responses induced by crude platycodin as well as by glyceryl trinitrate, papaverine or Saponin, the mechanism through muscarinic, histamine-like or beta-adrenergic effect may be ruled out from the point of vasodilating activity. Crude platycodin appears to have a direct vasodilating activity on the blood vessels as in the case of glyceryl trinitrate or papaverine, although more detailed experiments on the mechanism involved in the vasodilating effect are required for confirmation.

Acknowledgements: The authors are indebted to Prof. S. Shibata and Dr. T. Akiyama, Faculty of Pharmaceutical Sciences, University of Tokyo, and Prof. O. Tanaka, School of Medicine, Hiroshima University, for kindly providing crude platycodin.

\section{REFERENCES}

1) Aktyama, T., Tavaka, O. And Shibata, S.: Chem. Pharm. Bull. 20, 1945 (1972)

2) TAKAGi, K. AND Lli, E.B.: Yakigaka Zasshi 92, 951 (1972) (in Japanese)

3) TaKAGI, K. AND LeE, E.B.: Yakugaku Zasshi 92, 961 (1972) (in Japanese)

4) TakaGil, K. AND LeF, E.B.: Yakugaku Zasshi 92, 969 (1972) (in Japanese)

5) Kawashima, K., I.fF, E.B., Hirat, T., Takelchi, K. and Takagi, K.: Chrm. Pharm. Bull. 20, 755 (1972)

6) L.t, E.B.: Yakugaku 7asshi 93, 1182 (1973) (in Japancse) 